\title{
Ipsilateral Lateral Suture Suspension Platysmaplasty: A Modified Less Invasive Technique in Neck
}

\author{
NADA A. MAHMOUD, M.D. and KARIM S. MASSOUD, M.D. \\ The Department of Plastic, Burn and Maxillofacial Surgery, Faculty of Medicine, Ain Shams University, Cairo, Egypt
}

\begin{abstract}
Background: Numerous techniques have been described for neck lifting aiming at minimal access and limited downtime. This study presents a surgical technique for patients with considerable neck bands avoiding the anterior neck incision.

Patients and Methods: Thirteen female patients (mean age 57 years) with considerable platysma laxity underwent suture platysmaplasty during face lift procedures. Through a face lift incision, the platysma was suspended to the ipsilateral mastoid periosteum using permanent suture. The outcome was rated according to a modified visual analogue scale for neck bands and cervicomental angle.

Results: Twelve patients achieved satisfactory stable improvement. One case had early recurrence of her platysma bands that required surgical correction.

Conclusion: Modified ipsilateral suture suspension platysmaplasty, through the facelift approach can improve platysma bands without the need for additional anterior approach. This modified technique makes the procedure easier and less invasive.
\end{abstract}

Evidence Based Medicine: Level IV, case series.

Keywords: Platysma bands - Face lift - Neck lift - Neck rejuvenation - Suture suspension.

\section{INTRODUCTION}

The continuous refinements in face lift techniques yielded to procedures with increasing complexity. Newer trends are directed for simple and minimal surgical procedures [1].

Most patients seeking for facial rejuvenation usually want to improve their appearance, rather than to look very young. Therefore, addressing the jowls and platysmal bands can give them satisfactory results. To achieve these goals, short scar facelift techniques were developed and often termed as "mini" facelifts. However, these short scar facelift techniques have limitations especially for the anterior neck area [2].
In the 1990s, Vince Giampapa and Di Bernardo introduced the concept of the suture suspension for neck lift. This technique gave a long-lasting effect for neck rejuvenation $[3,4]$. Our technique is a modification of the original suspension technique making it simpler and less invasive. We use a permanent suture to suspend the platysma to the ipsilateral mastoid fascia; this suture is done through the same short scar facelift incision without additional sub mental incision.

\section{PATIENTS AND METHODS}

Between March 2015 and June 2017, thirteen adult female patients underwent lateral SMASectomy with ipsilateral suture suspension platysmaplasty. They had mild to moderate jowls, poorly defined cervicomental angle, and considerable platysma laxity which was partially corrected by the clinical simulation test described by Labbe and colleges [5] Fig. (1). Patients with severe platysmal laxity were excluded from the study.

Pre-operative and post-operative photos were taken to all patients in frontal and lateral views.

\section{Pre-operative preparation:}

Patients requested to stop aspirin and aspirin products intake for 10 days pre-operatively. Also, patients were requested to hold smoking 2 weeks pre-operatively and post-operatively. All patients had a stable psychological condition and realistic expectation. The benefits and possible drawbacks were discussed, and an informed consent were obtained. Routine pre-operative investigations were also done.

\section{Surgical marking:}

Incision line was marked horizontally under the sideburns then extended to the preauricular area, and in the front of ear lobe. It re-ascended 
parallel to the retroauricular line then curved to follow the line of hair implantation Fig. (2).

\section{Anesthesia:}

The procedure was done under local anesthesia and I.V. sedation (midazolam $0.050 .1 \mathrm{mg} / \mathrm{kg}$ ). The face and neck were infiltrated with $0.5 \%$ lidocaine with epinephrine (1:4 00.000), through use of a 22 -gauge spinal needle. All procedures were done by the same surgical team.

\section{Technique (Fig. 3):}

Incision line was opened, and skin flap was elevated with careful hemostasis. The SMAS excision was marked as a rectangle Fig. (3A). The superior limb was located $1 \mathrm{~cm}$ below the zygomatic arch and the long axes were orientated parallel to the nasolabial fold, with an average width of 2-3 $\mathrm{cm}$. The rectangle extended for $4-5 \mathrm{~cm}$ below the jaw line into the neck.

Blunt dissection of the SMAS flap started from the lateral edge of the drawn rectangle at the medial margin of the parotid gland. Dissection extended to the medial border of zygomaticus major muscle medially and superiorly. Inferiorly, dissection continued below the jaw line (about $4 \mathrm{~cm}$ from the midline of the neck). The marked rectangular part of the SMAS was excised Fig. (3B).

The vector of SMAS pull was superolateral; elevating the inferomedial corner of the medial
SMAS line after excision in a superolateral direction. Closure of the remaining SMAS defect was carried out which defined the jaw line and improved nasolabial folds Fig. (3C).

The remaining redundancy in the anterior neck region was addressed by the suspension suture avoiding an additional submental incision Fig. (4). The suture anchored the medial border of the platysma to the mastoid periosteum. The suture was taken at the desired level of the new cervicomental angle; usually at the level of hyoid bone nearly $1-2 \mathrm{~cm}$ below the angle of the mandible. We used a permanent Prolene suture 2-0 for women and 0 for men.

The suture was done as a figure of eight through the platysma. The suture was tied carefully avoiding any tethering or dimpling of the overlying skin while the patient's face was turned toward the other side and extended and excess skin redraped. This suspension suture was taken bilaterally to decrease the midline redundancy and improve cervicomental angle Fig. (1E). After tying of both sides, reassessment of the cosmetic results was done to ensure smooth contour and symmetry of both sides of the neck.

Finally, the cervicofacial flap was re-draped and closed after excision of redundant skin in the preauricular and retroauricular areas. A drain was inserted and passed out in the posterior hairline incision.

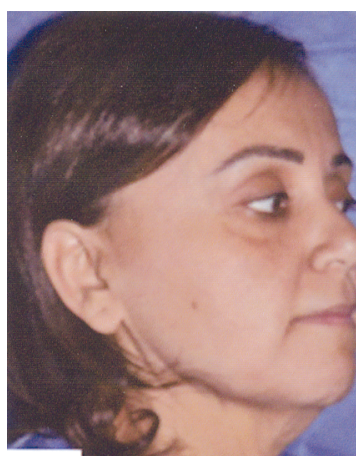

(A)

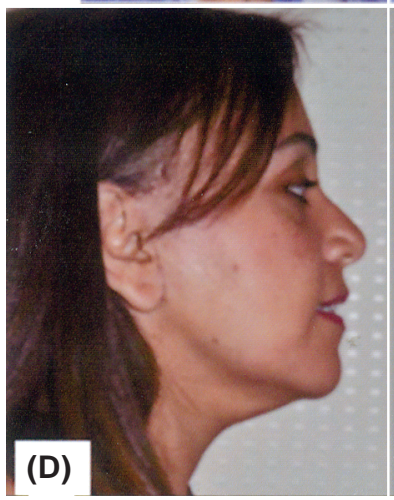

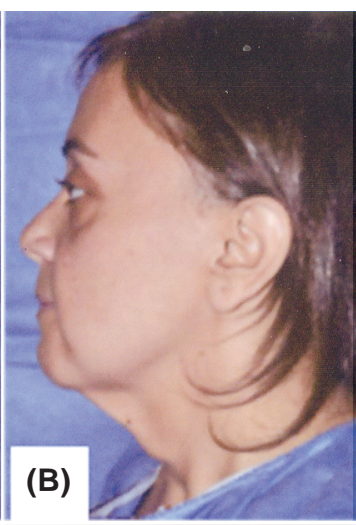

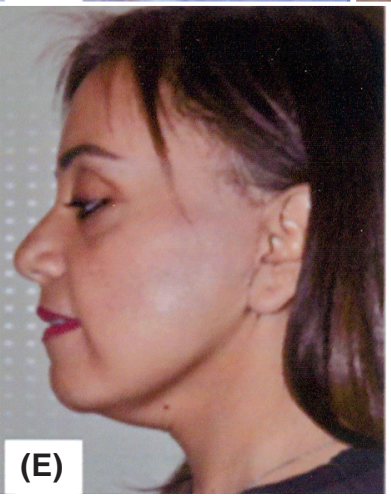

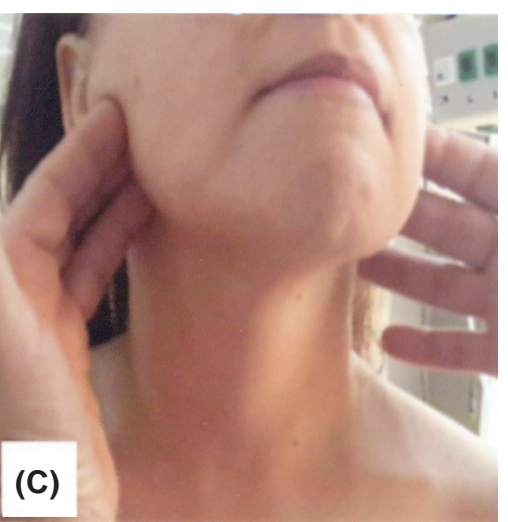

Fig. (1): (A,B): Pre-operative views of a 52 year old female patient, (C): Examination of neck laxity with clinical simulation test, $(\mathrm{D}, \mathrm{E})$ : Early post-operative views. 
Fig. (2): (A,B): Anterior and lateral views showing the preoperative markings for areas of suspected dissection and excess skin.
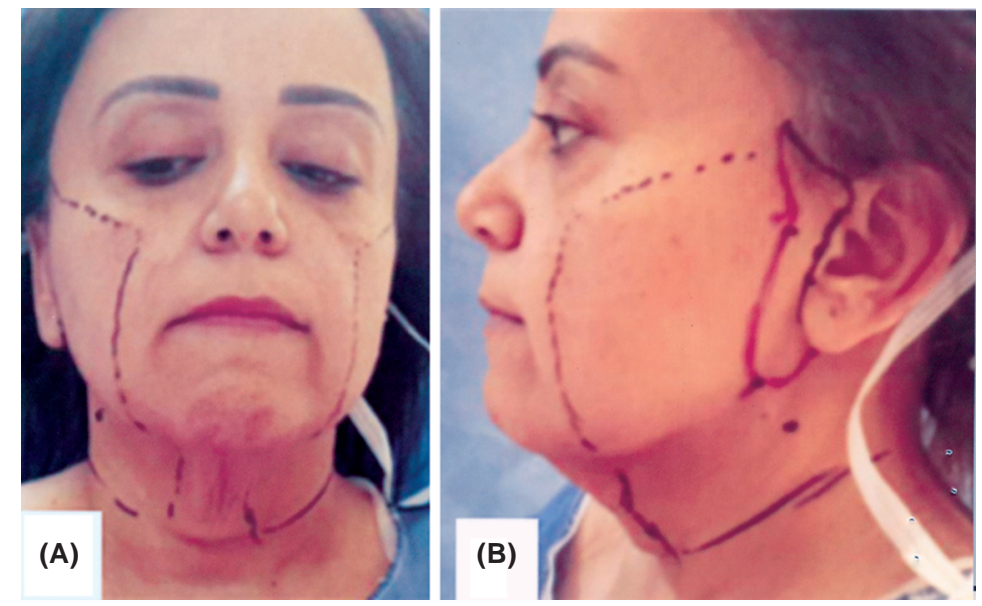

Fig. (3): (A) Intraoperative preauricular and postauricular skin incision, (B) Marking of the excised SMAS extending from below the zygomatic arch to below the jaw line parallel to the nasolabial fold, (C) Closure of SMAS after SMASectomy.
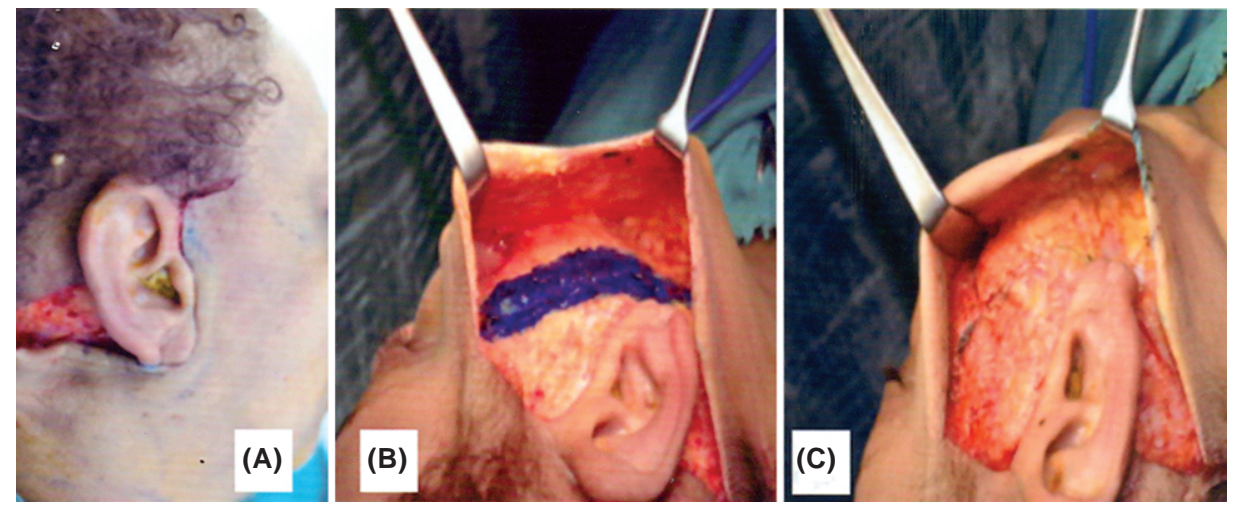

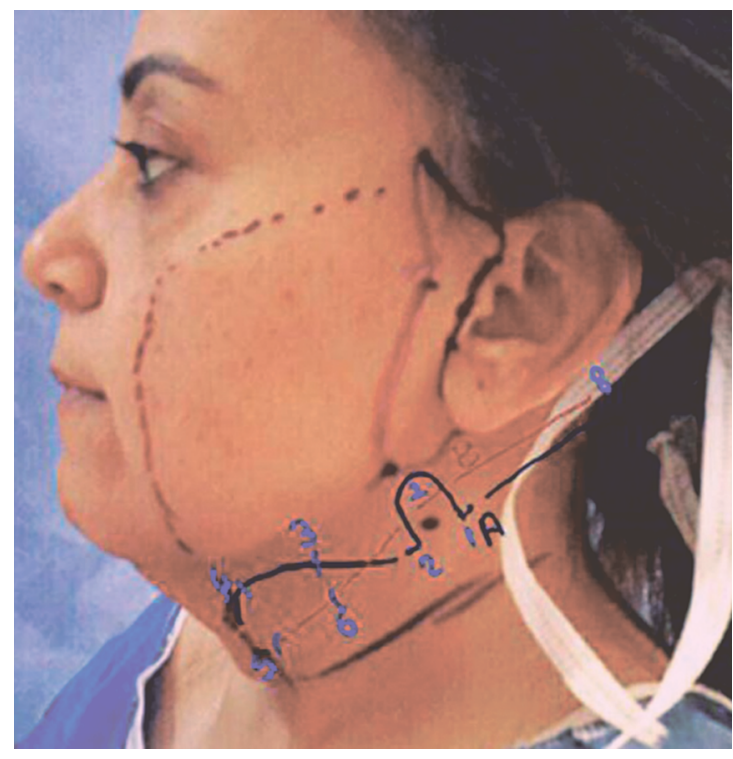

Fig. (4): Modified suture suspension technique, point 1 is below the angle of mandible acting as anchoring point that transfer the pull from horizontal to vertical vector. It is taken as a loop and not tied. Points 2-4 represents horizontal mattress suture. Points 4-5 is a vertical mattress suture at the anterior border of platysma. Points 5-6 represents the horizontal mattress returning back suture. The suture passes through the loop between points 1 and 2 . Then, the suture changes from horizontal to vertical direction and secured to the center of the mastoid fascia including periosteum.

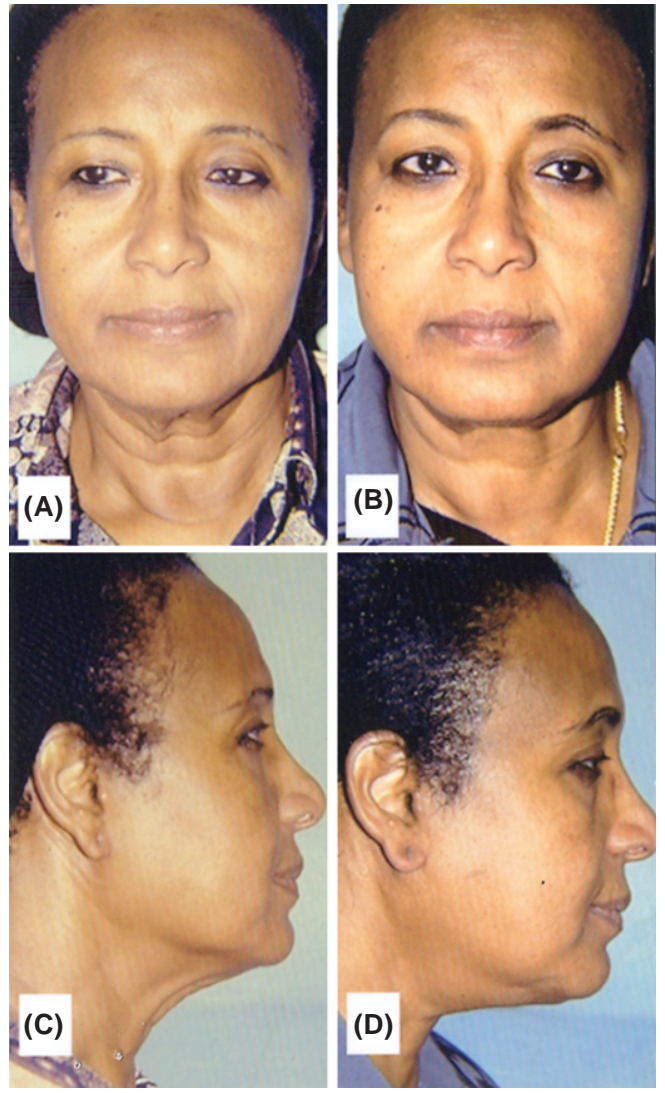

Fig. (5): A 54-year-old woman (A,B) Frontal pre and postoperative views. (C,D) Lateral profile pre and post-operative views 18 months later. 
Post-operative:

Patients received oral antibiotics, analgesics, and anti-inflammatory medications for one week (antibiotics for 3 days only). Dressings and the paper tape were removed after 48 hours. The patient discharged home next day, and stitches removed after 1 week. Follow-up visits were scheduled every 2 weeks in the first month then every 3 months. Post-operative photos were obtained each visit. The patients filled their satisfaction level in each visit.

Assessment of results were reviewed clinically and photographically by an independent surgeon unfamiliar with the patients and blinded to the procedure. The results were calculated as a mean of the records by the surgical team, the patient herself, and an independent surgeon. We used a visual analog scale for assessment; the scale rated the degree of improvement of neck laxity including neck banding, and the cervicomental angle [6] (Table 1).

\section{RESULTS}

Patients' age ranged from 49 to 67 years with a mean of 57.2 years. Three of them were hyper- tensive and medically controlled. Post-operative course passed unevenly in all cases except for transient edema in the first 1-2 two weeks. No hematoma or facial nerve injuries were recorded.

Twelve patients had satisfactory outcome with the results of neck lift and platysmaplasty. This satisfactory outcome was recorded by both patients' satisfaction and surgeon satisfaction. One patient showed early recurrence of platysma bands. This patient required anterior approach platysmaplasty after 6 months. All the thirteen patients showed good results regarding the lateral SMASectomy for mid-face lifting. The degree of improvement of cervicomental angle was moderate improvement with a mean of 5.02. The results of neck banding improvement showed moderate improvement with a mean of 4.1 (Table 1).

Neither hematomas nor seromas were recorded. No flap necrosis either partial or total loss occurred. No wound infection or dehiscence were found. No prolonged neck tightness was recorded. Tightness by the suture usually occurred in the first 3 months but later decreased then disappeared in about 6 months.

Table (1): Visual analogue scale for assessment of patients' outcome and our patients' scores.

\begin{tabular}{|c|c|c|c|c|c|c|}
\hline \multicolumn{6}{|l|}{ Visual analogue scale } & \multirow{2}{*}{$\begin{array}{c}\text { Our patients scores } \\
4.1(\mathrm{SD} 0.90)\end{array}$} \\
\hline $\begin{array}{cc}\text { Cervicomental angle: } \\
1 & 2 \\
\text { None } & \text { Mild }\end{array}$ & $\begin{array}{c}3 \\
\text { Improv }\end{array}$ & $\begin{array}{c}4 \\
\text { Mod }\end{array}$ & $\begin{array}{c}5 \\
\text { Improv }\end{array}$ & $\begin{array}{c}6 \\
\text { Dramatic }\end{array}$ & $\begin{array}{c}7 \\
\text { Improv }\end{array}$ & \\
\hline $\begin{array}{cc}\text { Neck banding: } \\
1 & 2 \\
\text { None } & \text { Mild }\end{array}$ & $\begin{array}{c}3 \\
\text { Improv }\end{array}$ & $\begin{array}{c}4 \\
\text { Mod }\end{array}$ & $\begin{array}{c}5 \\
\text { Improv }\end{array}$ & $\begin{array}{c}6 \\
\text { Nearly }\end{array}$ & $\begin{array}{c}7 \\
\text { Effaced }\end{array}$ & $5.02(\mathrm{SD} 0.90)$ \\
\hline
\end{tabular}

\section{DISCUSSION}

The aim of any face lifting procedures are to obtain a satisfactory improvement with the least possible scars. Baker [7] started short scar facelift using SMASectomy technique and achieved excellent results especially in younger patients. However, in older patients with cervical skin laxity and platysmal banding, he clearly recognized the limitations of the technique regarding anterior neck rejuvenation. Berend and co-workers [2] stated that visible platysma bands cannot be solved without a platysmaplasty procedure.

The manipulation of the platysma muscles in the neck rejuvenation was initially published in 1970s by Guerrero-Santos and colleagues [8] then Connell [9]. Feldman [10] published the corset platysmaplasty. Later, many techniques followed achieving the goals through resections, transections, partial transections, suture lifting, suture slinging, and plication approaches [11-13]. However, recurrence of platysma laxity following this diversity of techniques was common [14].

Giampapa and Di Bernardo [3] introduced the suture suspension technique for neck lift. They used it first in patients with open facelift, then performed as a neck lift approach.

The suture platysmaplasty can exert many effects in neck rejuvenation. First, the suspension suture helps to elevate the platysma muscle into its new position. This in turn improves the cervicomental angle and mandibular angle definition. In addition, improvement of neck bands helps to correct anterior neck width. It helps to define the neck from the cheek and support ptotic submandibular gland [11]. 
Suture suspension has many advantages such as short recovery downtime, less incidence of soft tissue or nerve injuries, and hidden postauricular scar. It allows neck lifting without a facelift which is good option for males. When combined with facelift (as in our study), it can give shorter downtime. However, it is not recommended for thin patients, because it may result in surface irregularity or it can be palpable [15].

Labbé and colleges [5] recommended platysma suspension only for lateral bands over the submaxillary gland and the Sternocleidomastoid muscle. On the other hand, they recommended corset platysmaplasty for the medial bands over the digastric muscle area.

The modifications that we added to the suture platysmaplasty technique make the technique simpler and faster. It eliminates the need for anterior neck incision which helps in reduced morbidity and shorter downtime which is an important issue in aesthetic patients. The figure of 8 design gives better traction and prevents the suture to cut through muscle fibers. Our work shows that both medial and lateral bands can be corrected using the modified ipsilateral suture lifting. The suture loop at angle of mandible acts as a vector as described by Giampapa $[\mathbf{3 , 4 , 1 5}]$.

The mid-face lifting and Submuscular Aponeurotic System (SMAS) manipulation must be done before the neck lifting because the SMAS is pulled together with the jaw fatty tissue. If the reverse is done, the fat can be raised too high and create a higher jawline than desirable [16]. From technical point of view, SMASectomy extends below jawline; so it is done first before suture suspension to avoid cutting the suture during SMASectomy.

In our work, we added the ipsilateral suture suspension of the platysma (including the medial and lateral bands) toward the mastoid process during mid-face lifting. This suture suspension is done through the already available pre-auricular facelift incision without additional submental incision. Our results showed moderate improvement of the cervicomental angle and neck bands. This modified technique made the procedure shorter, less invasive, and easier.

\section{Conclusion:}

In conclusion, combining the lateral SMASectomy with ipsilateral suture suspension platysmaplasty can address all signs of neck laxity without additional anterior neck incision. The ipsilateral suspension makes the procedure easier, less invasive, and shorter.

\section{REFERENCES}

1- Tonnard P., Verpaele A., Monstrey S., et al.: Minimal access cranial suspension lift: A modified S-lift. Plast. Reconstr. Surg., 109: 2074, 2002.

2- Berend van der Lei, M.D., Ph.D.; Michel Cromheecke, M.D., Ph.D. and Stefan O.P. Hofer, M.D., Ph.D.: The Purse-String Reinforced SMASectomy Short Scar Facelift. Aesthetic Surg. J., 29: 180-8, 2009.

3- Giampapa V.C. and Di Bernardo B.E.: Neck recontouring with suture suspension and liposuction: An alternative for the early rhytidectomy candidate. Aesthetic Plast. Surg., 19: 217-23, 1995.

4- Giampapa V., Bitzos I., Ramirez O., et al.: Long-term results of suture suspension platysmaplasty for neck rejuvenation: A 13-year follow-up evaluation. Aesthetic Plast. Surg., 29: 332-40, 2005.

5- Labbé D., Giot J. and Kaluzinski E.: Submental Area Rejuvenation by Digastric Corset: Anatomical Study and Clinical Application in 20 Cases. Aesth. Plast. Surg., 37: 222-31, Doi 10.1007/s00266-013-0083-7, 2013.

6- Adamson P., Dahiya R. and Litner J.: Midface Effects of the Deep-Plane vs the Superficial Musculoaponeurotic System Plication Face-lift. Arch. Facial Plast. Surg., 9: 9-11, 2007.

7- Baker D.C.: Minimal incision rhytidectomy (short scar facelift) with Lateral SMASectomy: Evolution and application. Aesthetic Surg. J., 21: 14-26, 2001.

8- Guerrero-Santos J., Espaillat L. and Morales F.: Muscular lift in cervical rhytidoplasty. Plast. Reconstr. Surg., 54: 127-30, 1974.

9- Connell B.F.: Contouring the neck in rhytidectomy by lipectomy and muscle sling. Plast. Reconstr. Surg., 61: 376-83, 1978.

10- Feldman J.J.: Corset platysmaplasty. Clin. Plast. Surg., 19: 369-82, 1992.

11 - Roerich R.J., Rios J.L., Smith P.D. and Gutowski K.A.: Neck rejuvenation revisited. Plast. Reconstr. Surg., 118: 1251-63, 2006.

12- Tonnard P.L. and Verpaele A.M.: The MACS-lift short scar rthytidectomy. Quality Medical Publishing, St. Louis, 2004.

13- Marten T.J.: High SMAS facelift: Combined single flap lifting of the jawline, cheek and midface. Clin. Plast. Surg., 35 (4): 569-603, 2008.

14- Hodgkinson D.: Five-Year Experience with Modified Fogli (Lore"s Fascia Fixation) Platysmaplasty. Aesth. Plast. Surg., 36: 28-40, Doi: 10.1007/s00266 011-97722,2012 .

15- Giampapa V. and Mesa J.: Neck Rejuvenation with Suture Suspension Platysmaplasty Technique A Minimally Invasive Neck Lift Technique That Addresses All Patients' Anatomic Needs. Clin. Plastic Surg., 41: 109-24, http:// dx.doi.org/10.1016/j.cps.2013.09.005, 2014.

16- Gonzalez R.: The LOPP-Lateral Overlapping Plication of the Platysma An Effective Neck Lift Without Submental Incision. Clin. Plastic Surg., 41: 65-72, http:// dx.doi.org/10.1016/j.cps.2013.09.015, 2014. 